\title{
Pathology, molecular mechanisms and markers of gliomas: new insight and new challenges
}

\author{
Werner Paulus ${ }^{1}$
}

Published online: 12 May 2015

(C) Springer-Verlag Berlin Heidelberg 2015

In the last years we have seen tremendous progress in the understanding of the molecular pathology of the major groups of brain tumors, in particular gliomas and medulloblastomas. MGMT promoter methylation, IDH mutation, BRAF mutations, and loss of $1 \mathrm{p}$ and $19 \mathrm{q}$ as the most prominent genetic changes play major roles in everyday counseling of glioma patients, and many other genetic aberrations are emerging at different stages from bench to bedside. There is no doubt that molecular data already play major roles in the diagnosis, classification, therapy and prognosis of brain tumors, and virtually nobody in the field questions the necessity of complementing histological phenotypes with genotypes. The long-standing vision that detailed molecular analysis and classification of brain tumors will result in personalized, more effective therapy has become closer. However, we have to realize that we are still at the initial stages of molecular diagnostics and targeted therapy, and that a couple of hurdles need to be overcome.

This issue of Acta Neuropathologica includes a cluster of expert review articles on the current status of molecular neuropathology, mechanisms and molecular markers of gliomas, including pilocytic astrocytoma, diffuse astrocytoma, oligodendroglioma and glioblastoma. The papers review the state of the art and discuss the challenges lying ahead. Problems and open questions are remarkably similar among the major glioma types. How should we classify and treat tumors where histology and molecular pathology do not correspond,

Werner Paulus

paulusw@uni-muenster.de

1 Institute of Neuropathology, University Hospital Münster, Pottkamp 2, 48149 Münster, Germany such as oligodendrogliomas showing the classical honeycomb pattern but lacking loss of $1 \mathrm{p}$ and $19 \mathrm{q}$ and IDH mutation? Does oligoastrocytoma and glioblastoma with oligodendroglial component really exist based on current genetic evidence? Which molecular markers should be routinely assessed in gliomas, and are there diagnostic algorithms? Which methods should be used for assessing IDH mutations and 1p/19 loss? It is generally believed and reiterated that molecular analysis is more objective than histology resulting in higher interrater reliability, but are there really good data available in support of this notion? Are criteria for histological grading valid for all molecularly defined subgroups of a histologically defined tumor type, such as diffuse astrocytoma with versus without IDH mutation (see the research paper by Reuss et al. which is also included in this issue)? Recent studies have convincingly demonstrated that pediatric diffuse and pilocytic astrocytomas, oligodendrogliomas and glioblastomas are molecularly and often prognostically distinct from their adult counterparts, but how should tumors be classified in the absence of hallmark mutations, such as diffuse astrocytoma without aberrations in IDH1/IDH2, MYB/MYBL1, $B R A F$ and FGFRl arising in a child? What is the role of non-invasive molecular diagnostic methods, such as liquid biopsy (see the review paper by Best et al. in this issue)? These tantalizing questions and many others are addressed in this issue.

This is an exciting time for molecular neuro-oncology with important discoveries being made at an ever increasing pace during the past and the upcoming few years. It is hoped that the papers of the glioma cluster will be informative, stimulating and thought-provoking for the newcomer as well as for the experienced basic and clinical neuro-oncologist. 